\title{
Perceptually-Motivated Coded Apertures
}

\author{
B. Masia, L. Presa, D. Gutierrez \\ Graphics and Imaging Lab-Advanced Computer Graphics Group (GIGA) \\ Instituto de Investigación en Ingeniería de Aragón (I3A) \\ Universidad de Zaragoza, Mariano Esquillor s/n, 50018, Zaragoza, Spain. \\ Tel. +34-976762707, Fax +34-976762043, e-mail: bmasia@unizar.es
}

\begin{abstract}
Computational perception techniques are gaining increasing importance in all different steps of the imaging pipeline, from data capture to final display. The goal of these techniques is usually the same: to minimize visible errors in the final image. We present a method to compute coded apertures for defocus deblurring that takes into account human perception to obtain the optimal aperture pattern. We pose the problem of finding the optimal aperture as an optimization, and to our knowledge- propose the first algorithm that makes use of perceptual quality metrics in its objective function. We explore the performance of different quality metrics for the design of such coded apertures, including the well-established SSIM, the state-of-the-art HDR-VDP-2, which features a comprehensive model of the HVS, as well as the L2 norm, previously used in related works.

Our results outperform both conventional circular apertures and a previously published aperture pattern specifically designed for defocus deblurring [1], both in simulated and real scenarios (see Figures 1 and 2). The best apertures are obtained when a combination of the three metrics is used in the objective function. This work has been recently accepted for publication at the Computer Graphics Forum [2].
\end{abstract}
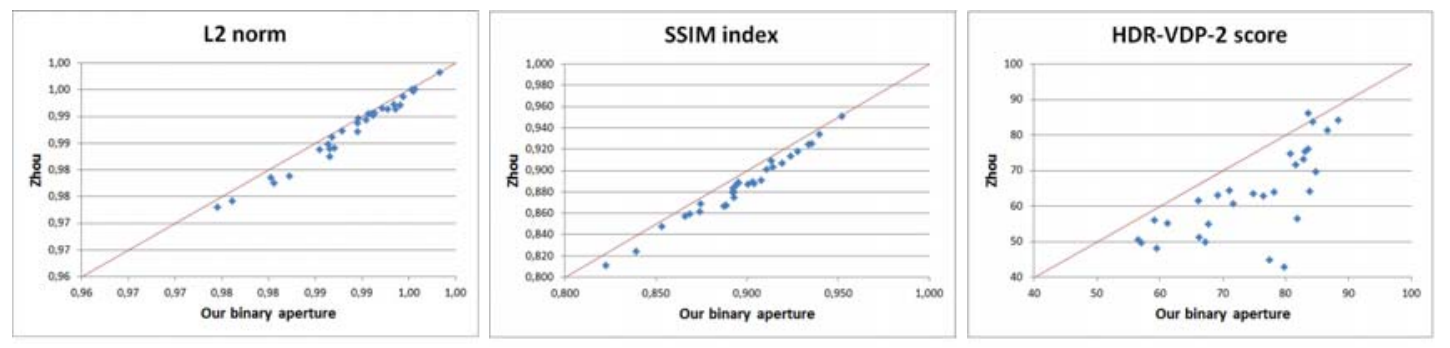

Figure 1: Scatter plots showing the performance of our best binary coded aperture against the coded aperture proposed by Zhou et al. [1] for an image noise of $\sigma=0: 005$. The L2 norm is depicted as (1-L2/100), L2 being the percentage with respect to the maximum error.
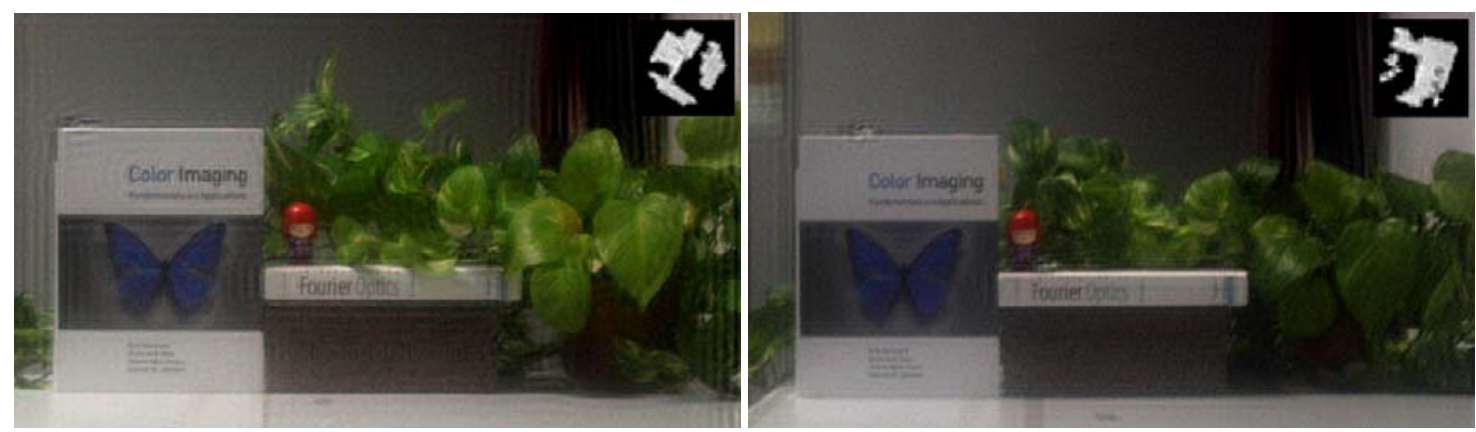

Figure 2: Recovered images captured with the aperture proposed by Zhou et al. [1] (left), and with our best perceptually-optimized binary aperture (right). Note the drastic reduction of ringing artifacts. 